\title{
MCM5 Expression Is Associated With the Grade of Malignancy and Ki-67 Antigen in LSCC
}

\author{
KATARZYNA NOWINSKA ${ }^{1}$, URSZULA CIESIELSKA ${ }^{1}$, ALEKSANDRA PIOTROWSKA ${ }^{1}$, \\ KAROLINA JABLONSKA ${ }^{1}$, ALEKSANDRA PARTYNSKA ${ }^{1}$, MARIA PAPROCKA ${ }^{2}$, \\ TOMASZ ZATONSKI ${ }^{3}$, MARZENA PODHORSKA-OKOLOW ${ }^{4}$ and PIOTR DZIEGIEL ${ }^{1,5}$ \\ ${ }^{1}$ Department of Human Morphology and Embryology, Division of Histology and \\ Embryology, Wroclaw Medical University, Wroclaw, Poland; \\ ${ }^{2}$ Laboratory of Cellular Interactions, Ludwik Hirszfeld Institute of Immunology and \\ Experimental Therapy, Polish Academy of Sciences, Wroclaw, Poland; \\ ${ }^{3}$ Department of Otolaryngology, Head \& Neck Surgery, Wroclaw Medical University, Wroclaw, Poland; \\ ${ }^{4}$ Division of Ultrastructure Research, Wroclaw Medical University, Wroclaw, Poland; \\ ${ }^{5}$ Department of Physiotherapy, Wroclaw University School of Physical Education, Wroclaw, Poland
}

\begin{abstract}
Background/Aim: The minichromosome maintenance proteins (MCMs) may be potential biomarkers of cancer cell proliferation. They are essential to initiate DNA replication. The aim of the study was to investigate the level of MCM5 expression in benign lesions (BLs) and laryngeal squamous cell cancer (LSCC). Materials and Methods: Immunohistochemical (IHC) analysis was carried out on 83 LSCCs and 10 BLs. Western-blot, immunofluorescence analysis $(I F)$ and real-time PCR (RT-PCR) were performed using HEp-2 cancer cells and HaCaT keratinocytes. Results: The expression of MCM5 was higher in LSCC than in the BLs $(p<0.0001)$ and was higher in subsequent malignancies of LSCC. Positive correlations were demonstrated between the expression levels of MCM5 and the Ki-67 antigen. In vitro studies have confirmed that the expression of MCM5 is elevated in cancer cells. Conclusion: MCM5 protein may be used as a potential marker of cancer cell proliferation in LSCC.
\end{abstract}

Laryngeal carcinoma is a very common type of cancer of the upper respiratory tract, which leads to a decreased quality of life in patients throughout Europe. Laryngeal squamous cell cancer (LSCC) is the most frequent histological type.

This article is freely accessible online.

Correspondence to: Katarzyna Nowinska, Ph.D., Department of Human Morphology and Embryology, Division of Histology and Embryology, Wroclaw Medical University, Chalbinskiego 6a, 50365 Wroclaw, Poland. Tel: +48 717841354, Fax: +48 717840082, e-mail: katarzyna.nowinska@umed.wroc.pl

Key Words: MCM, minichromosome proteins, Ki-67, laryngeal squamous cell cancer.
Despite a slight decrease in morbidity and mortality in the last 10 years in most European countries, it still remains a serious medical problem (1). Therefore, it is necessary to find new prognostic and predictive markers for LSCC. Numerous studies on other types of cancers have indicated that minichromosome maintenance proteins (MCMs) may be potential markers of malignant transformation of cells (2).

The MCM proteins are essential to initiate DNA replication. Duplication of DNA is a multistep process that involves the formation of replication forks and it is strictly controlled to coordinate DNA synthesis with the cell cycle and cell energy level. During DNA replication over 50 different protein factors are recruited to the site of the initiation of DNA replication throughout the genome. One of the most important and wellknown factors is the MCM2-7 complex, which is a helical helix composed of 6 subunits of different MCM proteins. At the MCM2/5 unit, there is a gap which allows the entry of the DNA strand into the ring shape complex of MCM2-7 $(3,4)$. This complex contributes to the formation of replication forks and determines where DNA duplication will proceed. The close connection of MCM2-7 with origins of DNA replications is accomplished by additional factors such as the origin recognition complex (ORC), Cdc6 and Cdt1. The MCM2-7 complex acts also as a limiting factor for DNA replication only once during the entire cell cycle. It is attached to the DNA replication origin in the $\mathrm{G}_{1}$ phase of the cell cycle and bound to the DNA during the $S$ phase (5-7). Expression of MCM2-7 is detected in the nucleus only in dividing cells. However, it is not observed in the interphase during differentiation or in ageing cells (8).

The association between the overexpression of MCMs with the clinicopathological factors and progression in various types of cancers has been confirmed in several studies (9-15). However, there are few studies that have investigated the 
expression levels of MCM5 and their relationship with the clinicopathological factors in tumours (13-17). MCM5 overexpression is associated with larger tumour size, lymph node metastasis and greater progression in oral squamous cell carcinoma (15). The elevated level of MCM5 expression correlates with a higher grade of malignancy and a worse prognosis in gastric adenocarcinomas (16), with more advanced malignancy in bladder cancer (14), ovarian adenocarcinoma (13) and skin cancer (17). So far, the expression of MCM proteins and their association with clinicopathological data in laryngeal cancer have been poorly understood. However, increased expression of MCM2 has been observed in $\operatorname{LSCC}(8,18,19)$.

Considering the contribution of MCM proteins to cell proliferation, it seems reasonable to compare their expression levels with the Ki-67 antigen, a well-established cell proliferation marker used in the diagnosis of proliferating tumour cells. It is a non-histone protein present in the nucleus of dividing cells (20). In LSCC, the increase in the $\mathrm{Ki}-67$ proliferation index is associated with progression and greater tumour aggressiveness $(8,21,22)$. To the best of our knowledge, this is the first study examining the relationship of Metallothionein I and II (MT-I/II) with MCM5 in LSCC. Metallothioneins are proteins that can bind zinc and copper ions necessary for cell functioning (23). $\mathrm{Zn}$ ions are necessary for enzyme activity and therefore MT-I/II might influence the activity of multiple factors controlling DNA replication, transcription and translation. It is also believed that MT-I/II are involved in the control of cell proliferation and differentiation (24). MT-I/II showed a mean positive correlation with MCM3 protein in LSCC (8).

As yet no studies have been conducted on the expression of MCM5 protein in LSCC. Therefore, the levels of MCM5 expression in benign lesions and LSCC were investigated using immunohistochemistry (IHC). The association of MCM5 with the clinicopathological factors and its value as a prognostic marker in LSCC were also analysed.

\section{Materials and Methods}

Patients. The study material consisted of 83 paraffin blocks with LSCC sections. Fifty one blocks were obtained from the Department of Pathomorphology of the J. Babiński Provincial Hospital in Wroclaw and 32 cases from the Department and Clinic of Otolaryngology, Head and Neck Surgery, Wroclaw Medical University. The patients were diagnosed and treated at the Department of Laryngology from 1997 to 2003. Ten sample blocks of benign lesions (BLs) of the laryngeal epithelium were used as a control (vocal cord nodules and Reinke's oedema). The study was approved by the Bioethical Commission at the Wroclaw Medical University (ID No. KB-343/2012). All patients gave informed consent for the use of tissues and the processing of disease data. The mean age of patients in the group was 60 years (range $=39-79$ years). The treatment and follow-up group consisted of 12 women and 71 men. The grade of malignancy $(\mathrm{G})$ and the clinical stage of LSCC were based on the TNM classification determined by the
International Union Against Cancer (UICC). The clinical characteristics of the patient group are summarised in Table I and have been presented previously (8).

Cell line culture. Immunofluorescence (IF) and western blot experiments were performed with the use of the reference adherent laryngeal cancer cell line, larynx epidermoid carcinoma HEp-2 and immortal adult human keratinocyte against normal human keratinocyte cell line $\mathrm{HaCaT}$ which was used as a control (collection of cell lines of the Ludwik Hirszfeld Institute of Immunology and Experimental Therapy, Polish Academy of Sciences, Wroclaw, Poland). The HEp-2 cells were cultured in EMEM medium (Lonza, Basel, Switzerland) and HaCaT cells were cultured in DMEM medium (Lonza) supplemented with $10 \%$ foetal bovine serum, (FBS) (Merck, Darmstadt, Germany), 1\% penicillin/streptomycin (Merck) and L-glutamine (Merck). The HERA cell (Heraeus, Hanau, Germany) incubator was used to culture cells under constant conditions i.e. temperature of $37^{\circ} \mathrm{C}, 5 \% \mathrm{CO}_{2}$ concentration and a $95 \%$ humidity level.

Immunohistochemistry reaction (IHC). The tissue sections were fixed in $4 \%$ buffered formalin, dehydrated and embedded in paraffin. In each case, hematoxylin and eosin staining was performed for the verification of histopathology by two independent pathologists. The paraffin blocks were freshly cut into paraffin sections of $4 \mu \mathrm{m}$ thick. Deparaffinization, hydration and thermal epitope demasking were performed for $20 \mathrm{~min}$. by boiling at $97^{\circ} \mathrm{C}$ in Dako PT Link (Dako, Glostrup, Denmark) high-pH Target Retrieval Solution. To evaluate the antigen expression in LSCC and BLs, IHC was performed using specific monoclonal mouse antibodies i.e. anti-MCM5 (E-10 clone, 1:100 dilution, Santa Cruz Biotechnology, Dallas, TX, USA) anti-Ki-67, anti-MCM2, 3, 7 or anti-MT-I/II. They were used and performed according to Nowinska et al. (8). The antibodies were diluted in background-reducing reagent and the incubation with sections was carried out for $1 \mathrm{~h}$ at room temperature. All IHC reactions were conducted with the use of Autostainer Link 48 (Dako) apparatus and EnVision ${ }^{\mathrm{TM}}$ FLEX reagents (Dako) visualization system. IHC reaction without the use of the primary antibody was performed as a negative control.

Evaluation of immunohistochemistry reactions. The expression levels of the tested proteins were analysed by two independent investigators at magnification of $\times 200$ with the use of a BX41 (Olympus, Tokyo, Japan) light microscope coupled with a DP-12 camera and CellD (Olympus) software for computer image analysis. The intensity of MCM5 expression was determined with the use of a five-point evaluation scale (score $0-4$ ), considering the percentage of cancer cells exhibiting reaction relative to all cancer cells in a given specimen (Table II) (25). However, MCM2, 3, 7 and Ki-67 and MT-I/II were evaluated according to Nowinska et al. (8).

Double immunofluorescence (IF) reaction. In order to perform double IF reaction, 24-h microcultures of laryngeal cancer cells and normal human keratinocytes were set up on 8-well Teflon-covered slides. For microculture inoculum, $50 \mu \mathrm{l}$ of $5 \times 10^{4}$ cells $/ \mathrm{ml}$ suspension in the appropriate medium was instilled into the wells on the slide. Slidemicrocultures were placed on Petri dishes in a cell incubator (HERA, Heraeus) at $37^{\circ} \mathrm{C}, 5 \% \mathrm{CO}_{2}$ and $95 \%$ humidity for $24 \mathrm{~h}$. After the incubation time, the medium was removed and the slides were washed with PBS. The fixation of cells in the slide wells was made with the use of $4 \%$ formaldehyde in PBS for $12 \mathrm{~min}$. at room temperature. 
Table I. Clinicopathological characteristics of LSCC patients related to MCM5 expression.

\begin{tabular}{lcccc}
\hline $\begin{array}{l}\text { Clinicopathological } \\
\text { parameter }\end{array}$ & $\begin{array}{c}\mathrm{N} \\
83(\%)\end{array}$ & $\begin{array}{c}\text { MCM5 } \\
\text { low } \\
\text { expression } \\
0-2\end{array}$ & $\begin{array}{c}\text { MCM5 } \\
\text { high } \\
\text { expression } \\
3-4\end{array}$ & $\begin{array}{c}\text { Fisher's } \\
\text { exact } \\
\text { test } \\
p \text {-Value }\end{array}$ \\
\hline Gender & & & & \\
$\quad$ Male & $71(85.5 \%)$ & $39(47 \%)$ & $32(38.6 \%)$ & 0.5344 \\
Female & $12(14.5 \%)$ & $5(6 \%)$ & $7(8.4)$ & \\
Age & & & & \\
$<60$ & $44(53 \%)$ & $22(26.5 \%)$ & $22(26.5 \%)$ & $\mathbf{0 . 0 3 1 0}$ \\
$\geq 60$ & $29(34.9 \%)$ & $22(26.5 \%)$ & $7(8.4 \%)$ & \\
Tumour size (T) & & & & \\
$\quad$ T1-2 & $14(16.9 \%)$ & $5(6 \%)$ & $9(10.8 \%)$ & 0.2400 \\
T3-4 & $69(83.1 \%)$ & $39(47 \%)$ & $30(36.2 \%)$ & \\
Lymph nodes (N) & & & & \\
$\quad$ N0 & $51(61.4 \%)$ & $29(34.9 \%)$ & $22(26.5 \%)$ & 0.4983 \\
N1-3 & $32(38.6 \%)$ & $15(18.1 \%)$ & $17(20.5 \%)$ & \\
Stage & & & & \\
I-II & $10(12 \%)$ & $5(6 \%)$ & $5(6 \%)$ & 1.000 \\
III-IV & $73(88 \%)$ & $39(47 \%)$ & $34(41 \%)$ & \\
Grade of & & & & \\
malignancy (G) & & & & \\
G1 & $23(27.7 \%)$ & $40(48.2 \%)$ & $28(33.7 \%)$ & $\mathbf{0 . 0 4 3 0}$ \\
G2-3 & $60(72.3 \%)$ & $4(4.8 \%)$ & $11(13.3 \%)$ & \\
\hline
\end{tabular}

Bold values denote statistical significance.

Permeabilization of the cell membrane was carried out with $0.2 \%$ Triton in PBS for $10 \mathrm{~min}$. at room temperature. For the double reaction a mix of two primary antibodies was used i.e. anti-MCM5 mouse monoclonal antibody (1:100, clone E-10, code no. sc-165994, Santa Cruz Biotechnology, USA, TX, Dallas) and anti-Ki-67 rabbit monoclonal antibody (1:200, clone H-300, code no. sc-15402, Santa Cruz Biotechnology). Overnight incubation with primary antibodies was performed at $4^{\circ} \mathrm{C}$. Subsequently, the slides were incubated for $1 \mathrm{~h}$ with a mix of two secondary antibodies i.e. donkey anti-mouse FITC (fluorescein isothiocyanate)-conjugated antibody (1:50; Jackson ImmunoResearch Laboratories, West Grove, PA, USA) and donkey anti-rabbit TRITC (fluorescein tetramethylrhodamine)-conjugated antibody (1:50; Jackson ImmunoResearch Laboratories) diluted in the reagent with background-reducing component (Dako). Preparations were mounted using Prolong mounting medium with DAPI (Invitrogen, Carlsbad, CA, USA). Slides were observed under a BX51 microscope (Olympus) coupled with CellF software (Olympus).

Western Blot analysis. After culturing, the HEp-2 and HaCaT cells were trypsinized, centrifuged and washed in PBS. The number of cells was estimated using Automated Cell Counter (Invitrogen). For each western blot analysis, $4 \times 10^{6}$ of laryngeal cancer cells and keratinocytes in the exponential growth phase were taken. Fractionation was performed with the NE-PER Nuclear and Cytoplasmic Extraction Kit (Thermo Scientific, Waltham, MA, USA) according to the manufacturer's protocol. The concentration of proteins was determined using a BCA Protein Assay Kit (Thermo Scientific). Equal amounts of nuclear proteins were denatured in the sample loading buffer $(250 \mathrm{mM}$ Tris- $\mathrm{HCl}, 40 \%$ glycerol, $20 \% \beta$ -
Table II. The scale assessing protein expression levels of MCM5 in benign lesions of the larynx and LSCC.

\begin{tabular}{lc}
\hline Point & The percentage of cells with positive reaction (\%) \\
\hline 0 & $0 \%$ \\
1 & $1 \%-10 \%$ \\
2 & $11 \%-25 \%$ \\
3 & $26 \%-50 \%$ \\
4 & $>51 \%$ \\
\hline
\end{tabular}

mercaptoethanol, $8 \%$ sodium dodecyl sulfate (SDS) and bromophenol blue) at $96^{\circ} \mathrm{C}$ for $10 \mathrm{~min}$. and subjected to SDS-PAGE. The proteins were transferred to PVDF membrane (Millipore, Burlington, MA, USA) and unspecific binding sites were blocked with 5\% non-fat milk (Bio-Rad, Hercules, CA, USA) in TBST for $1 \mathrm{~h}$ at room temperature. The membrane was incubated overnight at $4^{\circ} \mathrm{C}$ with anti-MCM5 mouse monoclonal antibody diluted in $0.5 \%$ milk in TBST (1:500, clone E-10, code no. sc-165994, Santa Cruz Biotechnology). Next, incubation with secondary horseradish peroxidase conjugated donkey anti-mouse antibody $(1: 3000$, code no. 715-035-150; Jackson ImmunoResearch) was performed for $1 \mathrm{~h}$ at room temperature. Proteins were visualised using Luminata Classico Western HRP Substrate (Millipore). The membrane was stripped and reprobed with anti-histone H3 mouse monoclonal antibody $(1: 1,000$, clone 96C10, code no. 3638S, Cell Signalling Technology, Leiden, The Netherlands), which was used as a control of protein amount loading. The data were collected in Chemi-Doc XRS Molecular Imager (Bio-Rad) apparatus. Optical density of the protein band was measured with the use of Image Lab (Bio-Rad) software. The experiment was repeated three times.

Real-time PCR analysis. The RNeasy Mini Kit was used (Qiagen, Hilden, Germany) for the isolation of RNA from HEp-2 and HaCaT cells. The reverse transcription reaction was performed using HighCapacity cDNA Reverse Transcription Kit with RNase Inhibitor (Applied Biosystems, Waltham, MA, USA). Changes in the MCM5 expression level (MCM5; TaqMan Gene Expression Assay, Applied Biosystems) were tested with using 7900HT Fast Real-Time PCR System (Applied Biosystems). Analysis of the MCM5 gene expression was performed using the relative quantification (RQ) method and RQ Manager 1.2 software (Applied Biosystems). The results were standardized, based on expression of the reference gene of $\beta$-actin (ACTB; TaqMan Gene Expression Assay, Applied Biosystems, Waltham, MA, USA). The changes in the levels of MCM5 gene expression in HEp-2 cells were tested in relation to HaCat cells. The evaluation of MCM5 gene expression by real-time PCR was repeated 3 times. The obtained results are shown in the graphs on a logarithmic scale and subjected to statistical analysis.

Statistical analysis. The Kolmogorov-Smirnov test was used to evaluate the normality assumption of the examined groups. The statistical significance of the differences in the expression of the tested proteins in benign lesions and laryngeal carcinomas was determined using a non-parametric equivalent of the $t$-Student test i.e. the $U$-Mann-Whitney test. Spearman's rank correlation was used to evaluate the relationship between the expression of the analysed 
MCM2, 3, 5 and 7 proteins as well as the Ki-67 antigen and MT-I/II However, the Kruskal-Wallis test was used to assess the correlation between the intensity of MCM5 protein expression and the degree of differentiation (G, G1- well, G2-moderately, G3-poorly) of the examined laryngeal tumours. Additionally, $U$-Mann-Whitney test was used for comparison between the MCM5 protein expression and the degree of malignance (G1 vs. G2, G2 vs. G3, G1 vs. G3). The Kaplan-Meier analysis and the Cox regression model were used to examine the relationship between the intensity of MCM5 expression and patient survival. The Fisher's exact test was used to evaluate the level of MCM5 expression in relation to clinicopathological parameters. The student $t$-test was used to evaluate the expression level of MCM5 between cancer cells and keratinocytes in in vitro studies. The statistical analysis was performed using Prism 5.0 software (GraphPad, La Jolla, CA, USA).

\section{Results}

Comparison of MCM5 expression levels in BLs and LSCC. IHC analysis indicated that MCMs and the Ki-67 antigen are localized in the nucleus and MT-I/II in the cytoplasm. The scale assessing protein expression level of MCM5 is presented in Table II. In BLs, the expression of MCM proteins, the Ki-67 antigen, and MT-I/II was detected only in the basal layer of the laryngeal epithelium and also in less than $50 \%$ of its height. The percentage expression of MCM5 proteins in BLs did not exceed the 2-point limit. Laryngeal cancers were characterised by high expression of the tested proteins. There were also cells where MCM5 expression was not observed (Figure 1).

However, higher expression of all tested MCM proteins, the Ki-67 antigen and MT-I/II was observed in cancers compared to BLs. The results of the U-Mann-Whitney test showed that the difference was statistically significant for all proteins. Data on MCM2, 3, 7 and Ki-67 and MT-I/II expression levels had been described in our previous study (8). The MCM5 expression level was lower in the group of BLs (mean=1.1 $\pm 0.32 \mathrm{SD}$ ) than in the subsequent LSCC malignancies (mean $=2.48 \pm 1.03 \mathrm{SD}$ ). The difference in the intensity of MCM5 expression between the groups was statistically significant $(p<0.0001)$ (Figure 2).

The associations of MCM5 expression levels with clinicopathological factors in LSCC. The clinicopathological characteristics of the patients in relation to the levels of MCM5 expression are presented in Table I. The correlation between the intensity of expression of the examined protein and the clinicopathological factors such as the grade of malignancy $(\mathrm{G})$, the presence of lymph node metastasis, tumour size and patient survival was analysed. The level of MCM5 expression in tumour cells was increasing with the increase in grade of malignancy of LSCC. A statistically significant difference was observed between the control group and subsequent malignancy (BL $v s$. G1 $p=0.0001$; BL vs. G2 $p<0.0001$; BL $v s . \mathrm{G} 3, p<0.0001)$, as well as between
G1 vs. G3 and G2 vs. G3 ( $p=0.0144, p=0.0298)$ (Figure 3A). MCM5 expression was the highest in T2 tumours (mean $=3 \pm 0.94 \mathrm{SD}$ ). In other tumour sizes, the mean values were between $2.25 \pm 1.1$ (T3) and $2.54 \pm 0.9$ (T4). A significant difference was observed between T2 and T3 in LSCC $(p=0.0173)$. However, Ki-67 expression was also higher in T2 (mean value $=2.50 \pm 0.3 \mathrm{SD}$ ) than in $\mathrm{T} 3$ tumours $(2.11 \pm 0.2)$. A correlation between MCM5 expression and lymph node metastases and stage of disease was not observed. Survival of LSCC patients was also analysed using the Mantel-Cox test. Patients with higher MCM5 expression in tumour cells showed a slightly shorter survival time compared to those with low protein levels. However, the difference was not statistically significant (Figure 3B). In the Cox regression model analysis only advanced $\mathrm{pN}$ status was related to shorter OS. The results of univariate analysis are presented in Table III.

The correlations of MCM5 expression levels with the other tested proteins in LSCC. Spearman's rank correlation analysis revealed a strong positive correlation between the expression of MCM proteins and the Ki-67 antigen in LSCC (Table IV). The correlations of MCM5 expression with the other MCM proteins and the Ki-67 antigen were statistically significant. No relationship was found between MCM5 and MT-I/II (Table IV).

Comparison of MCM5 expression levels in HEp-2 and HaCat cell lines. The IF analysis revealed expression of MCM5 and the Ki-67 antigen in the nuclei of cancer and normal cells. A double IF reaction detecting the presence of MCM5 and the Ki-67 antigen confirmed that the expression levels of both tested markers was higher in HEp-2 cancer cells compared to keratinocytes. Additionally, only some of the laryngeal cancer cells expressed both tested markers. Furthermore, some cells expressed only MCM5 while others only Ki-67 (Figure 4D). In order to semi-quantify expression of MCM5, western-blot analysis was performed in both tested cell lines. Statistical analysis of the densitometric measurements was carried out using the $t$-test. It revealed that the difference between the level of MCM5 expression in larynx HEp-2 cancer cells was statistically significant compared with keratinocytes $(p=0.0036)$. Moreover, Realtime PCR confirmed that the level of MCM5 mRNA expression is also higher in HEp-2 cells than in HaCat cells (Figure 4A-D).

\section{Discussion}

Cell proliferation proteins may be useful tumour biomarkers. Their usefulness is intensively studied as many of the mechanisms controlling tumour proliferation are disturbed during carcinogenesis. In most tumours, elevated 

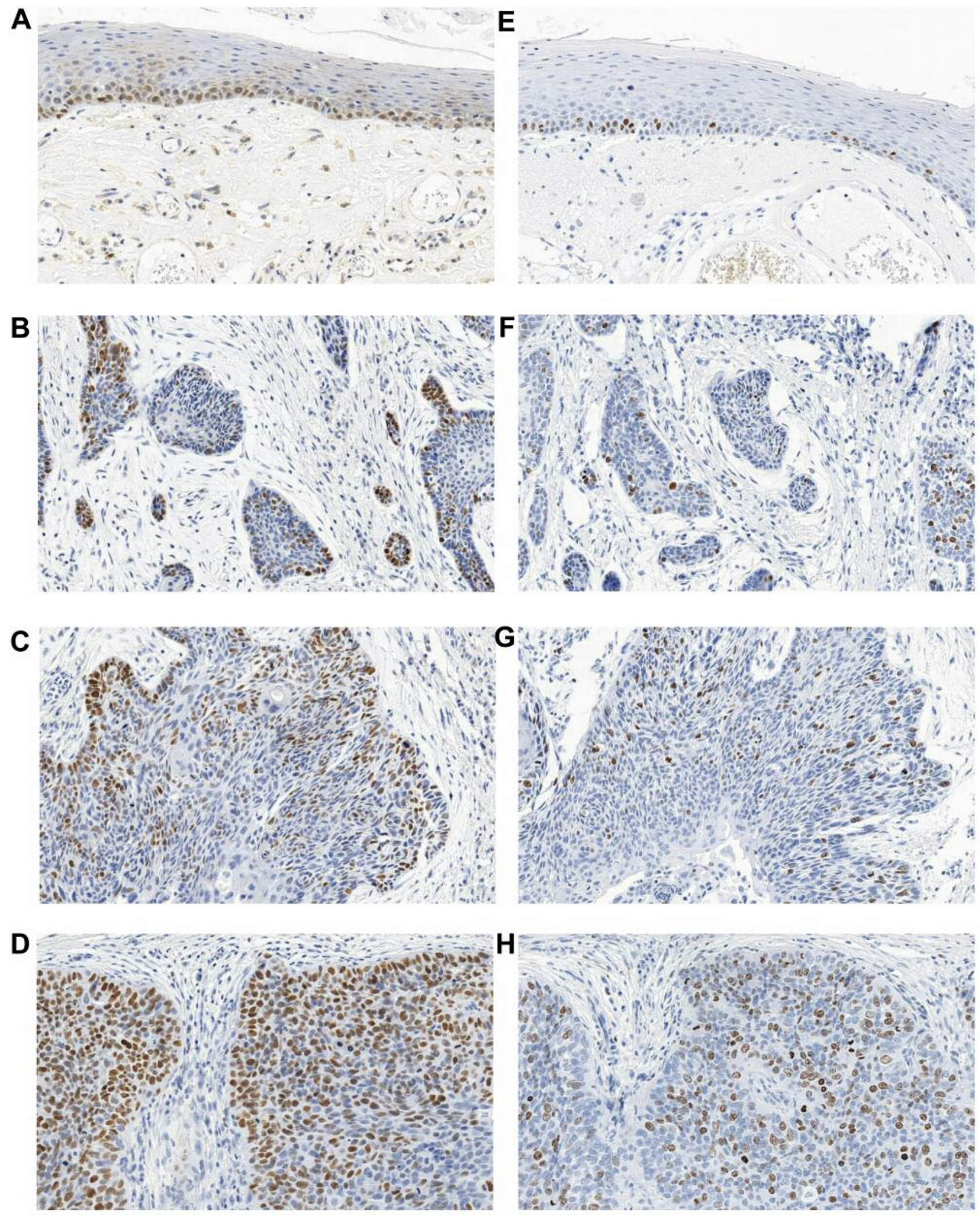

Figure 1. Positive immunohistochemical reaction (brown nuclei) indicating MCM5 (A-D) and Ki-67 antigen (E-H) expression carried out on BL and in different grades of LSCC malignancy. MCM5 expression - BL (A) and LSCC in G1 (B), in G2 (C) and in G3 (D). Ki-67 antigen expression in BL $(E)$ and LSCC in $G 1(F)$, in $G 2(G)$ and in $G 3(H)$, magnification, $\times 200$. 

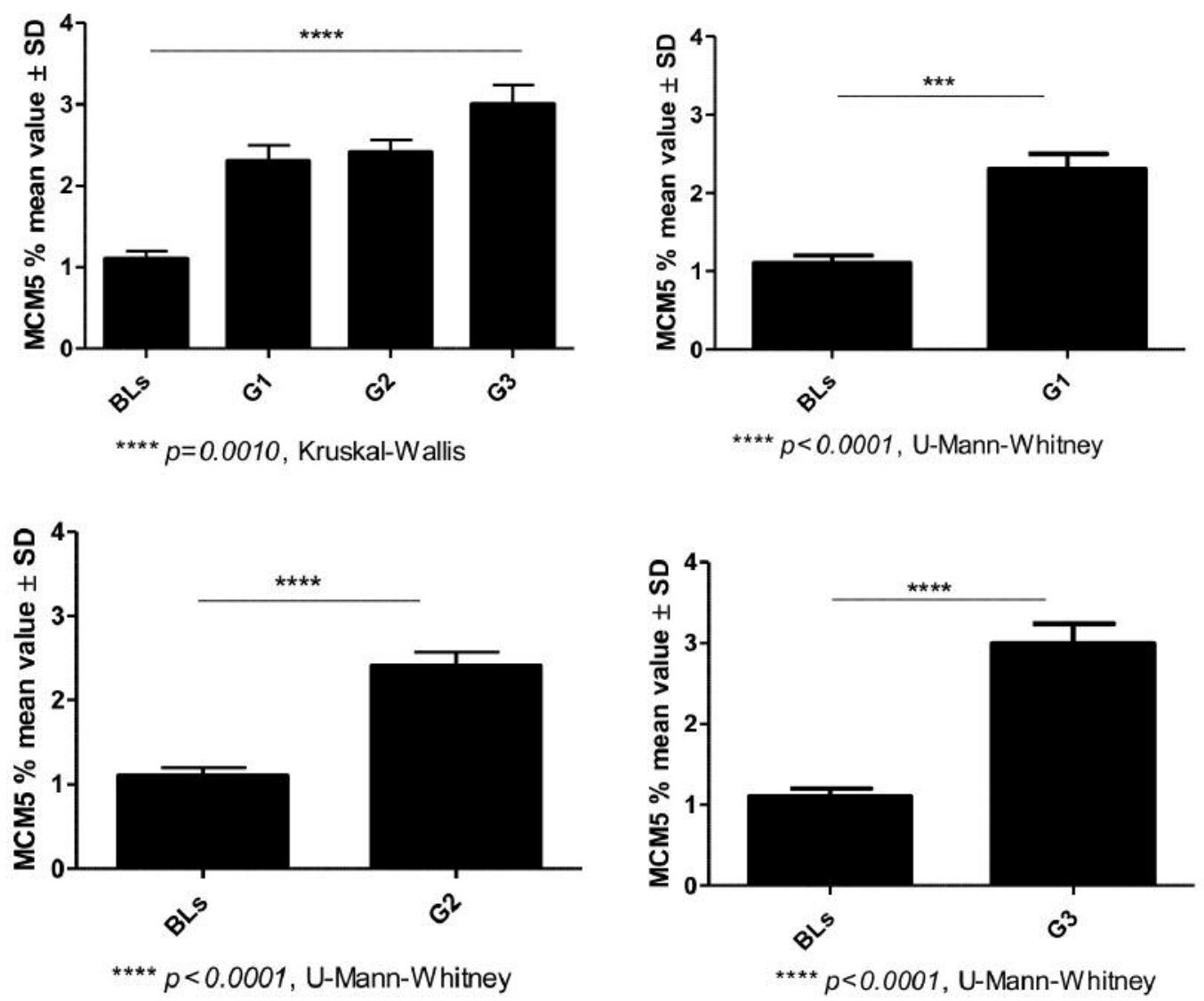

Figure 2. Comparison of MCM5 protein expression levels between BL and different grades of malignancy of LSCC.

expression levels of genes encoding proteins regulating cell proliferation is observed. The intensity of their expression may indicate rapid tumour growth and is often associated with a poorer prognosis $(5,26)$. MCMs are proteins that regulate cell proliferation. This group of proteins includes, among others, MCM5 whose levels of expression are first examined in the present study in LSCC and in BLs of the laryngeal epithelium. In our study, expression of MCM5 was observed only in the proliferating layer in the case of BLs. However, increased expression was observed in LSCC. Our studies are consistent with the results of other studies on other MCM proteins in normal and pathological conditions (16-19). Immunohistochemical investigation of the expression of MCM proteins in normal tissues demonstrated that their occurrence is limited only to the proliferating layers of the epidermal epithelium, intestinal mucosa and the lymphoid tissue. In turn, in pathological conditions the expression of MCMs was increased in precancerous lesions and solid tumours. The occurrence of MCM proteins in dysplastic and neoplastic cells may suggest their clinical usefulness in the diagnosis of preinvasive and invasive cancers $(5,27,28)$.
Chatrath et al. (19) have investigated the expression of MCM2 proteins and the Ki-67 antigen in 20 patients with laryngeal dysplasia and in 10 with LSCC. The obtained results were compared with the control group, consisting of 10 normal sections of laryngeal epithelium. They found that the expression of MCM2 proteins in the normal laryngeal tissue was low and limited only to the proliferating layer of the epithelium (19). Cai et al. (29) have also observed increased expression levels of both MCM2 and the Ki-67 antigen in LSCC as compared to normal laryngeal tissues and vocal cord polyps. Although the study of Chatrath et al. (19) is consistent with our results, it was conducted on a small study group, and only the expression of MCM2 and the Ki-67 antigen was compared with clinicopathological factors. However, the researchers did not verify the association between the expression of MCM5 and the clinical data. In the present study, increased expression of MCM5 and the Ki-67 antigen in 83 LSCC cases was observed compared to their expression in normal control tissues. A relationship between increased levels of MCM5 protein expression and the tumour size and histologic tumour grade score was also observed. 


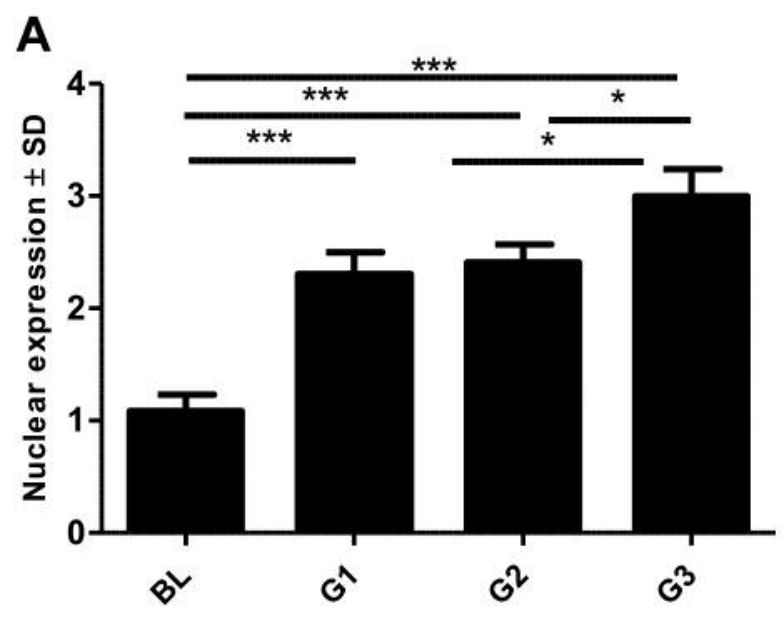

B

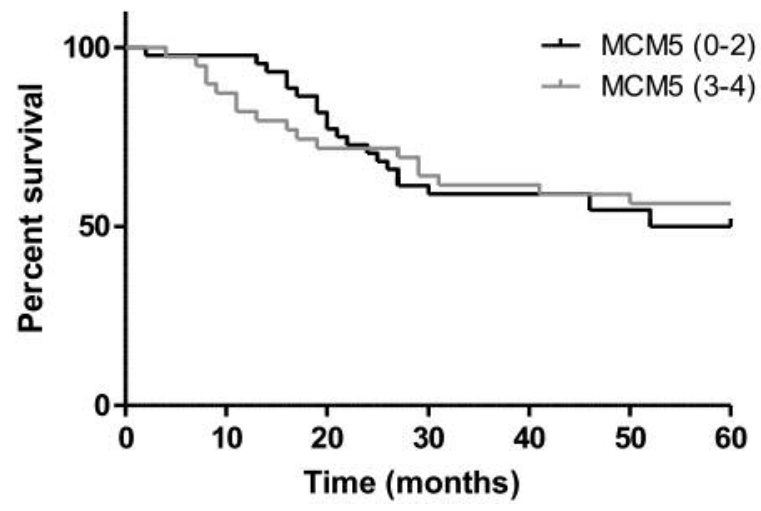

Figure 3. Comparison of MCM 5 protein expression levels in benign lesions and different grades of malignancy $(G)$ of LSCC. Significant differences in the values are marked $* * * p \leq 0.0001, * p<0.05$ (A). Comparison of patient survival percentage with low (0-2) and high (34) levels of MCM5 expression (Mentel-Cox analysis, $p=0.9921)(B)$.

Chartach et al. (19) have also performed a small pilot study on the presence of MCM5 protein expression in laryngeal brushings in 6 patients with laryngeal dysplasia. They observed expression of MCM2 and MCM5 proteins in patients who were diagnosed with laryngeal dysplasia. Similarly, Mukherjee et al. (30) have examined the usefulness of MCM2 and MCM5 protein expression in detecting abnormal cells in the superficial layer of the cervical epithelium. Cytological swabs were used to perform immunocytochemical staining. The Papanicolaou test (Pap) was compared with a test using anti-MCM antibodies. Pap staining is a routinely used method to diagnose altered cervical epithelial cells. The analysis performed with the Pap test is very subjective. If the material is taken incorrectly, false results can be obtained. Mukherjee et al. (30) have shown that immunocytochemical staining with the use of anti-MCM5 antibodies was a very specific and sensitive
Table III. Univariate Cox proportional hazards analysis in LSCC patients.

\begin{tabular}{lcc}
\hline & \multicolumn{2}{c}{ Univariate analysis } \\
\cline { 2 - 3 } & HR $(95 \% \mathrm{CI})$ & $p$-Value \\
\hline Age $(<60 v s . \geq 60)$ & $1.41(0.68-2.91)$ & 0.3475 \\
Gender (Male $v s$. Female) & $1.21(0.46-3.16)$ & 0.6912 \\
pT (T1-2 vs. T3-4) & $1.39(0.57-3.39)$ & 0.4642 \\
pN (N0 $v s$. N+) & $2.21(1.08-4.52)$ & $\mathbf{0 . 0 2 9 2}$ \\
Grade (G1 vs. G2-G3) & $1.89(0.77-4.61)$ & 0.1620 \\
Stage (I-II $v s$. III-IV) & $1.90(0.66-5.50)$ & 0.2330 \\
MCM5 (<25 \% vs. $\geq 25 \%)$ & $0.70(0.34-1.46)$ & 0.3484 \\
Necrosis $($ yes $v s$. no) & $0.62(0.36-1.29)$ & 0.2071 \\
Keratodes $v s$. akeratodes & $0.66(0.32-1.35)$ & 0.2599 \\
\hline
\end{tabular}

Bold values denote statistical significance.

Table IV. Spearman's rank correlation between the expression levels of MCM5 proteins and MCM2, 3, 7, Ki-67 antigen and MT-I/II in LSCC.

\begin{tabular}{lccccc}
\hline & Ki-67 & MT I/II & MCM 2 & $M C M 3$ & $M C M 7$ \\
\hline MCM5 & $\mathbf{r}=\mathbf{0 . 4 7}$ & $\mathrm{r}=0.13$ & $\mathbf{r}=\mathbf{0 . 5 7}$ & $\mathbf{r}=\mathbf{0 . 3 2}$ & $\mathbf{r}=\mathbf{0 . 4 1}$ \\
$p$-Value & $\boldsymbol{p}<\mathbf{0 . 0 0 0 1}$ & $p=0.2252$ & $\boldsymbol{p}<\mathbf{0 . 0 0 0 1}$ & $\boldsymbol{p}=\mathbf{0 . 0 0 4 0}$ & $\boldsymbol{p}=\mathbf{0 . 0 0 0 2}$ \\
\hline
\end{tabular}

Bold values denote statistical significance.

method enabling the detection of cervical cancers. The prognostic value of the MCM test was $100 \%$ for CIN1 and $79 \%$ for CIN3 stages (30). On the other hand, Stoeber et al. (31) have conducted a quantitative study using flow cytometry, aiming to develop a method for the detection of MCM5 in cells found in the urinary sediment. The study showed that such assays are sensitive, specific and allow early, simple and non-invasive detection of primary and recurrent tumours of the bladder. Flow cytometric detection of MCM5-positive cells proved to be a much more sensitive method in the diagnosis of neoplastic changes as compared to cytological tests of the urinary tract. The above studies confirm that among the MCM proteins, MCM5 could be useful for diagnostic purposes.

Previous studies on MCM proteins demonstrated that their expression was higher in cancer tissues compared with normal tissues, but also confirmed their relationship with the clinicopathological factors $(10,13,14,16,17,32-34)$. Our study also confirmed it. Our results revealed that the expression of MCM5 in LSCC was increased with the tumour growth, but only up to the T2 size. Also, the Ki-67 expression was the highest in T2. Perhaps in subsequent $\mathrm{T}$ sizes the rate of proliferation decreases. In the literature, no significant relationship between the levels of MCM5 expression and 
A
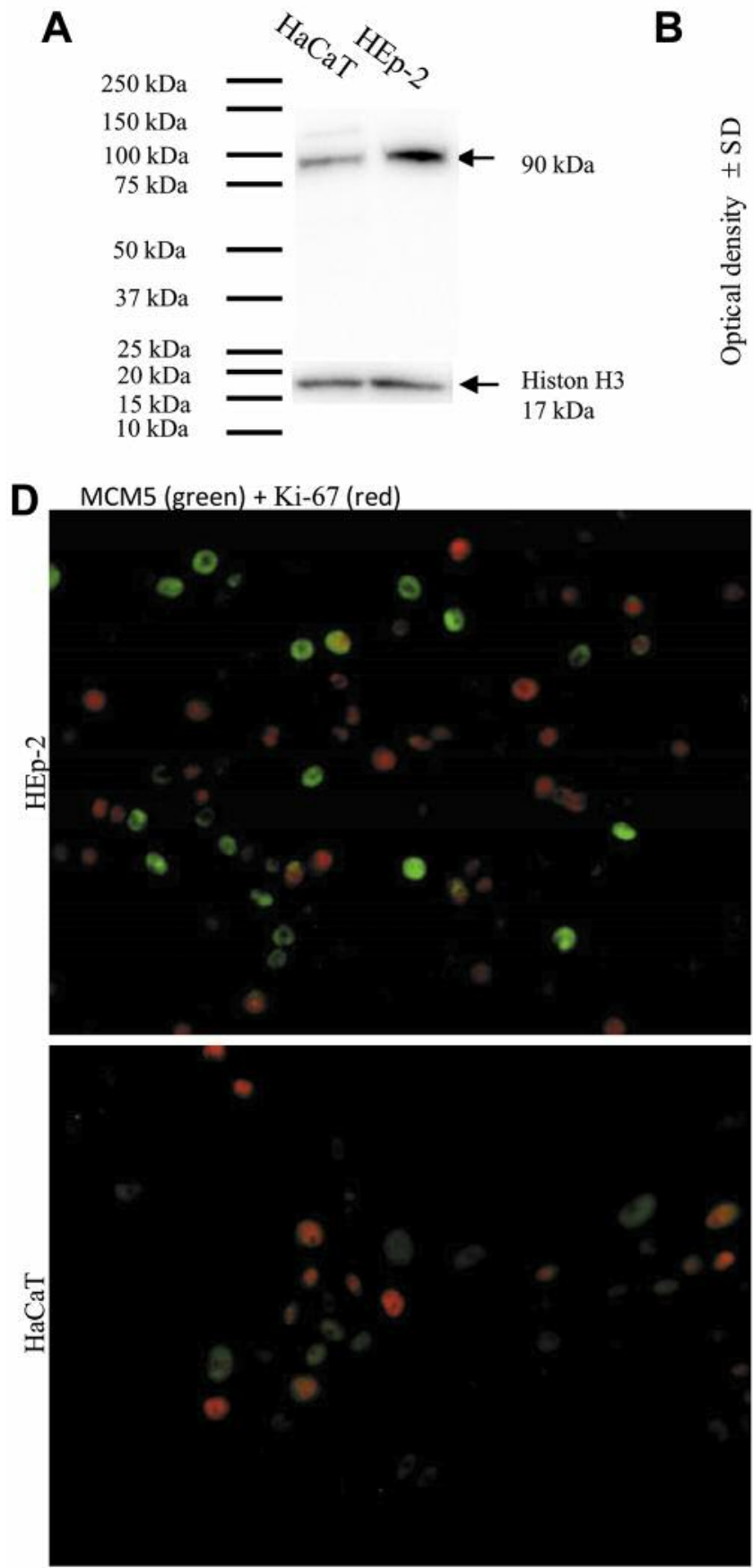

B

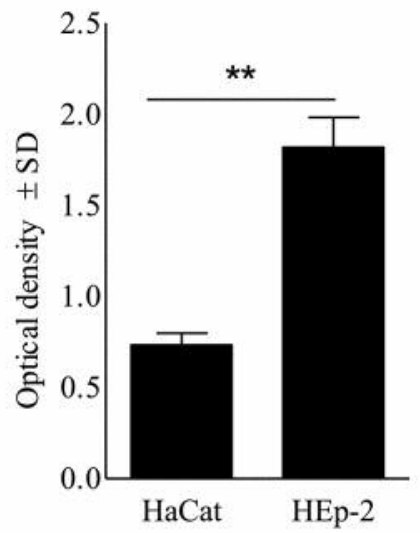

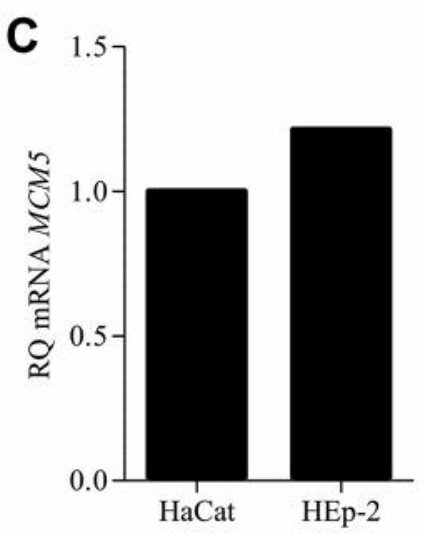

Merged
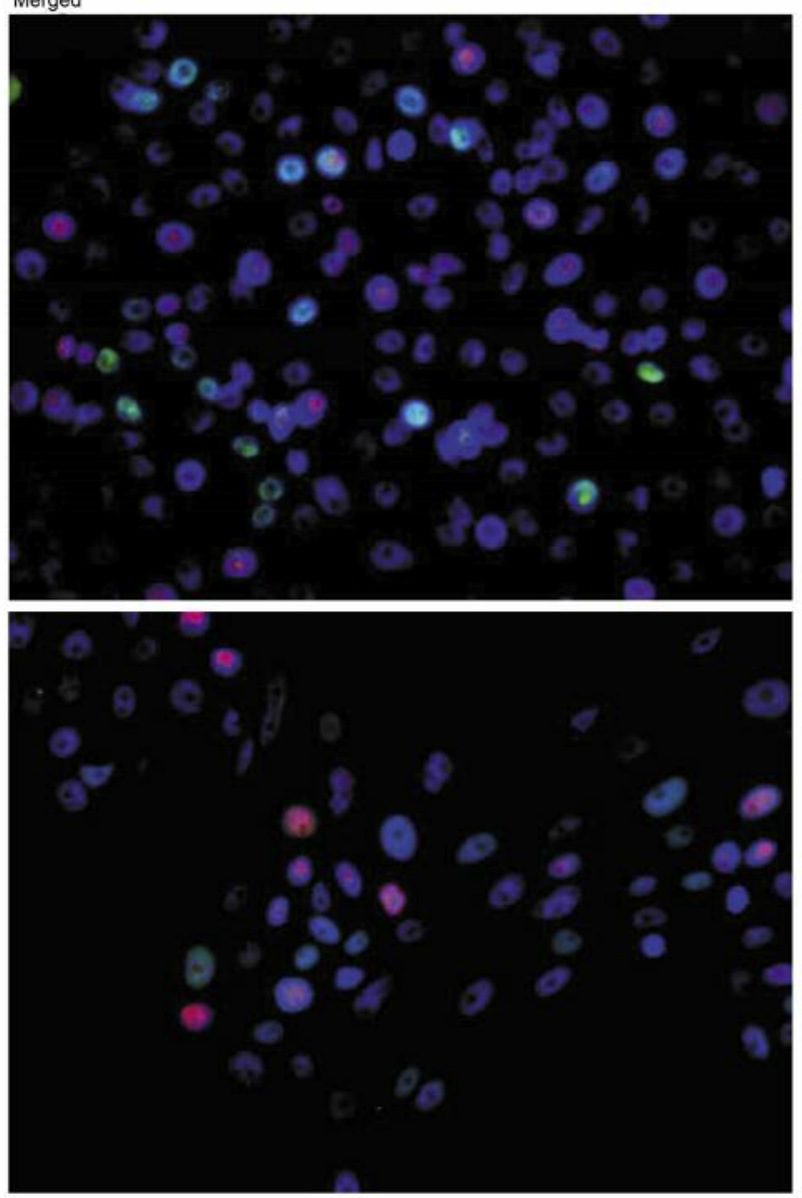

Figure 4. MCM5 (90 kDa) protein expression levels detected by using the western blot technique in the cell nucleus of HEp-2 cells and HaCat cells (A). Comparison of densitometric analysis of MCM5 protein expression levels between HEp-2 and HaCaT cells (**p=0.0036) (B). Comparison of mRNA MCM5 expression levels between HEp-2 and HaCaT cells $(C)$. Double immunofluorescence reaction detecting nuclear expression of MCM5 (green nuclei) and Ki-67 antigen (red nuclei) in HEp-2 cancer cells and normal human keratinocytes (HaCaT). Cell nuclei were visualized by DAPI dye (blue nuclei), magnification, $\times 400(D)$.

diverse tumour sizes (T) has been reported for LSCC. However, in studies on colon cancer, Giaginis et al. (16) have indicated that the expression of MCM2 correlated with selected clinicopathological parameters such as e.g. tumor size (T). In addition, in these studies, increased expression of MCM2 was also associated with lymph node metastases $(\mathrm{N})$ and the invasion of blood vessels. In our previous study (8) we have also shown that in the case of LSCC, the levels of MCM2 
expression were also increased in cancers with metastases to nearby lymph nodes. However, no statistically significant differences in the expression of other proteins i.e. MCM3 and MCM7 were observed. In the case of MCM5 in LSCC, no relationship with lymph node metastases was observed. Similarly, Han et al. (35) have examined samples of LSCC and did not notice a relationship between the expression level of MCM4 and lymph node metastases. Perhaps MCM2 is the only protein from the entire MCM2-7 complex that is associated with this clinicopathological factor. However, the comparison of the levels of expression and the degree of histological differentiation showed that the expression level of MCM5 was increased in subsequent grades of malignancy. Such an observation has also been reported for other types of cancer (13-17). On the other hand, no association with patient survival was found. Furthermore, no relationship between MCM5 and patient survival was observed, which is consistent with other studies $(16,36,37)$. Only a tendency was noticed indicating poor prognosis for patients with LSCC with increased expression levels of MCM5.

No correlation between MCM5 and MT-I/II was observed. According to our previous study, only MCM3 demonstrated an association with MT-I/II in LSCC (8). The reason for this may be the fact that MT-I/II can bind zinc and controls its level in the organism. From the entire MCM2-7 complex only MCM3 has a zinc binding motif. The MCM5 zincbinding motif is different than that in MCM3 (8). This could result in the lack of association between MCM5 and MT-I/II. Additionally, the level of MCM5 expression was strongly correlated with the level of expression of the approved diagnostic marker of proliferation (Ki-67 antigen) and other proteins i.e. MCM2, 3, and 7. More LSCC cells were revealed by examining the expression of MCM5 with IHC compared to the Ki-67 antigen and the dual labelling of IF detecting Ki-67 and MCM5 antibodies. The correlation between Ki-67 and other MCM proteins in both LSCC and other types of cancer has been confirmed $(13,16,19,33)$. Also, the relationship between MCM5 and other MCM proteins is predictable due to their participation in the formation of the MCM2-7 complex. Chartach et al. (18) have shown a correlation between Ki-67 and MCM2 protein expression in LSCC. Similarly to our study, using IHC, they observed that Ki-67 expression level was lower in LSCC compared to MCM2 proteins. Our results showed that only some of the laryngeal cancer cells expressed both tested markers. Some cells expressed only MCM5 while the others only Ki-67. Chartach et al. (18) have demonstrated that more cells showed expression of MCM proteins compared to the expression of Ki-67 in LSCC. This observation suggests that the Ki-67 antigen does not detect all proliferating cells or is less stable in the tissue than the MCM antigen in LSCC. However, it is also possible that MCMs could detect not only proliferative cells. Further studies are warranted to answer these questions. Although $\mathrm{Ki}-67$ is a standard proliferation marker, its cellular function has not been fully explained. It is known that this antigen localizes in the cell nucleus during mitosis. Phosphorylation of Ki-67 during mitosis is related to condensation of chromosomes, separation of sister chromatids and to the control of chromatin structure $(38,39)$. On the other hand, Endl et al. (40) have observed that growth-arrested cells, as confirmed by the absence of the Ki67 antigen, stained positive for the MCM3 protein. They indicated that the MCM3 protein was expressed in nonproliferating not terminally differentiated cells (40). MCM proteins are, however, directly associated with DNA replication and are present in the nucleus only during the division of the cell in all phases i.e. $\mathrm{G}_{1}, \mathrm{~S}, \mathrm{G}_{2}$ and M. MCM proteins become inactive when the cell leaves the division phase of the cell cycle and enters a quiescent or differentiation phase or when the cell is ageing. Loss of MCM activity occurs within a few days of entering a resting or differentiation phase. However, during the division cycle, MCM antigens are stable and detected using IHC (5). The lack of precise explanation of the Ki-67 function and the knowledge of its participation in ribosome biosynthesis are a disadvantage. On the other hand, a direct association of MCM proteins with DNA replication could indicate that MCMs may be useful additional diagnostic markers. The results of this and other studies confirmed the relationship between MCM proteins and cell proliferation and their potential diagnostic usefulness. Han et al. (35) have shown that silencing of the MCM4 gene by siRNA inhibited proliferation of UMSCC 5 cells. This confirms the prospect of using MCM proteins as a cellular target in anti-cancer therapy and their usefulness as a marker for proliferation of LSCC cells. Our studies have also confirmed that the MCM5 protein could also be useful in determining the rate of tumour development.

\section{Conclusion}

Our results indicate MCM5 as a potential and additional marker of tumour cell proliferation due to its contribution in DNA replication, its elevated expression in LSCC, and its positive correlation with $\mathrm{Ki}-67$, the standard proliferation marker. Our observations are consistent with other studies and confirmed the association of MCM5 expression with clinicopathological factors. Additionally, it seems reasonable to further study the usefulness of MCM5 in cytological tests for the diagnosis of various types of cancer. MCM proteins, including MCM5, may also become a potential molecular target in anti-cancer therapy.

\section{Conflicts of Interest}

The Authors declare that they have no competing interests. 


\section{Authors' Contributions}

NK and DP conceived and designed the experiments; ZT collected material from patients PA performed the IHC reactions; NK analyzed the data $\mathrm{PaA}$ performed the western blot experiments; JK performed IF reactions; NK and CU wrote the manuscript MPO and DP reviewed and revised the manuscript. All Authors have read and approved the final manuscript.

\section{Acknowledgements}

The research was supported by Wroclaw Research Centre EIT+ under the project "Biotechnologies and advanced medical technologies" - BioMed (POIG.01.01.02-02-003/08) financed from the European Regional Development Fund (Operational Programme Innovative Economy, 1.1.2)".

\section{References}

1 Wierzbicka M, Winiarski P and Osuch-Wójcikiewicz E: The incidence of laryngeal cancer in Europe with special regard to Poland in the last 2 decades. Otolaryngol Pol 70: 16-21, 2017. PMID: 27387212. DOI: $10.5604 / 00306657.1202782$

2 Gonzalez MA, Tachibana KK, Laskey RA and Coleman N: Innovation - Control of DNA replication and its potential clinical exploitation. Nat Rev Cancer 5: 135-141, 2005. PMID: 15660109. DOI: $10.1038 / \mathrm{nrc} 1548$

3 Riera A, Barbon M, Noguchi Y, Reuter LM, Schneider S and Speck C: From structure to mechanism - understanding initiation of DNA replication. Genes Dev 31: 1073-1088, 2017. PMID: 28717046. DOI: 10.1101/gad.298232.117

4 Gardner NJ, Gillespie PJ, Carrington JT, Shanks EJ, McElroy SP, Haagensen EJ, Frearson JA, Woodland A and Blow JJ: The high-affinity interaction between ORC and DNA that is required for replication licensing is inhibited by 2-arylquinolin-4-amines. Cell Chem Biol 24: 981-992.e4, 2017. PMID: 28781123. DOI: 10.1016/j.chembiol.2017.06.019

5 Nowińska K and Dzięgiel P: The role of MCM proteins in cell proliferation and tumorigenesis. Postepy Hig Med Dosw 64: 627-635, 2010. PMID: 21160097.

6 Aparicio T, Guillou E, Coloma J, Montoya G and Méndez J: The human GINS complex associates with Cdc45 and MCM and is essential for DNA replication. Nucleic Acids Res 37: 2087-2095, 2009. PMID: 19223333. DOI: 10.1093/nar/gkp065

7 Sclafani RA and Holzen TM: Cell cycle regulation of DNA replication. Annu Rev Genet 41: 237-280, 2007. PMID: 17630848. DOI: 10.1146/annurev.genet.41.110306.130308

8 Nowinska K, Chmielewska M, Piotrowska A, Pula B, Pastuszewski W, Krecicki T, Podhorska-Okołow M, Zabel M and Dziegiel P: Correlation between levels of expression of minichromosome maintenance proteins, Ki-67 proliferation antigen and metallothionein I/II in laryngeal squamous cell cancer. Int J Oncol 48: 635-645, 2016. PMID: 26648405. DOI: 10.3892/ijo.2015.3273

9 Eissa S, Matboli $M$ and Shehata HH: MicroRNA-10b and minichromosome maintenance complex component 5 gene as prognostic biomarkers in breast cancer. Tumor Biol, 2015. PMID: 25596707. DOI: 10.1007/s13277-015-3090-2

10 Wojnar A, Kobierzycki C, Krolicka A, Pula B, PodhorskaOkolow M and Dziegiel P: Correlation of Ki-67 and MCM-2 proliferative marker expression with grade of histological malignancy (G) in ductal breast cancers. Folia Histochem Cytobiol 48: 442-446, 2010. PMID: 21097442. DOI: 10.2478/ v10042-010-0069-0

11 Wojnar A, Pula B, Piotrowska A, Jethon A, Kujawa K, Kobierzycki C, Rys J, Podhorska-Okolow M and Dziegiel P: Correlation of intensity of MT-I/II expression with Ki-67 and MCM-2 proteins in invasive ductal breast carcinoma. Anticancer Res 31: 3027-3033, 2011. PMID: 21868554.

12 Kobierzycki C, Pula B, Skiba M, Jablonska K, Latkowski K, Zabel M, Nowak-Markwitz E, Spaczynski M, Kedzia W, Podhorska-Okolow $\mathrm{M}$ and Dziegiel P: Comparison of minichromosome maintenance proteins (MCM-3, MCM-7) and metallothioneins (MT-I/II, MT-III) expression in relation to clinicopathological data in ovarian cancer. Anticancer Res 33: 5375-5384, 2013. PMID: 24324072.

13 Gakiopoulou H, Korkolopoulou P, Levidou G, Thymara I, Saetta A, Piperi C, Givalos N, Vassilopoulos I, Ventouri K, Tsenga A, Bamias A, Dimopoulos M-A, Agapitos E and Patsouris E: Minichromosome maintenance proteins 2 and 5 in non-benign epithelial ovarian tumours: relationship with cell cycle regulators and prognostic implications. Br J Cancer 97: 1124-1134, 2007. PMID: 17940502. DOI: 10.1038/sj.bjc.6603992

14 Korkolopoulou P, Givalos N, Saetta A, Goudopoulou A, Gakiopoulou H, Thymara I, Thomas-Tsagli E and Patsouris E: Minichromosome maintenance proteins 2 and 5 expression in muscle-invasive urothelial cancer: A multivariate survival study including proliferation markers and cell cycle regulators. Hum Pathol 36: 899-907, 2005. PMID: 16112007 DOI: 10.1016/ j.humpath.2005.06.008

15 Yu S, Wang Y, Chang JY and Shen W: Increased expression of MCM5 is significantly associated with aggressive progression and poor prognosis of oral squamous cell carcinoma. J Oral Pathol Med 43: 344-349, 2014. PMID: 24245508. DOI: 10.1111/ jop. 12134

16 Giaginis C, Georgiadou M, Dimakopoulou K, Tsourouflis G, Gatzidou E, Kouraklis G and Theocharis S: Clinical significance of MCM-2 and MCM-5 expression in colon cancer: Association with clinicopathological parameters and tumor proliferative capacity. Dig Dis Sci 54: 282-291, 2009. PMID: 18465232. DOI: $10.1007 / \mathrm{s} 10620-008-0305-\mathrm{z}$

17 Liu H, Takeuchi S, Moroi Y, Lin N, Urabe K, Kokuba H, Imafuku S, Dainichi T, Uchi H, Furue M and Tu Y: Expression of minichromosome maintenance 5 protein in proliferative and malignant skin diseases. Int J Dermatol 46: 1171-1176, 2007. PMID: 17988337. DOI: 10.1111/j.1365-4632.2007.03335.x

18 Chatrath P, Scott IS, Morris LS, Davies RJ, Bird K, Vowler SL and Coleman N: Immunohistochemical estimation of cell cycle phase in laryngeal neoplasia. Br J Cancer 95: 314-321, 2006. PMID: 16832409 . DOI: 10.1038/sj.bjc.6603262

19 Chatrath P, Scott IS, Morris LS, Davies RJ, Rushbrook SM, Bird K, Vowler SL, Grant JW, Saeed IT, Howard D, Laskey R and Coleman N: Aberrant expression of minichromosome maintenance protein-2 and Ki67 in laryngeal squamous epithelial lesions. Br J Cancer 89: 1048-1054, 2003. PMID: 12966424. DOI: $10.1038 /$ sj.bjc.6601234

20 Sarafoleanu D, Postelnicu V, Iosif C, Manea C and Sarafoleanu $\mathrm{C}$ : The role of p53, PCNA and $\mathrm{Ki}-67$ as outcome predictors in the treatment of laryngeal cancer. J Med Life 2: 219-226, 2009. PMID: 20108544. 
21 Zatonski T, Ciesielska U, Nowinska K, Ratajczak-Wielgomas K, Kobierzycki C, Pula B, Podhorska-Okolow M, Krecicki T and Dziegiel P: Expression of cell cycle-related proteins p16, p27, p53 and Ki-67 in HPV-positive and-negative samples of papillomas of the upper respiratory tract. Anticancer Res 36: 3917-3924, 2016. PMID: 27466494.

22 Ciesielska U, Zatonski T, Nowinska, Katarzyna, RatajczakWielgomas K, Grzegrzolka J, Piotrowska A, Olbromski M, Pula B and Podhorska-Okolow, Marzenna, Dziegiel P: Expression of Cell Cycle-related Proteins p16, p27 and Ki-67 Proliferating Marker in Laryngeal Squamous Cell Carcinomas and in Laryngeal Papillomas. Anticancer Res 2415: 2407-2415, 2017. PMID: 28476808. DOI: 10.21873/anticanres.11580

23 Romero-Isart N, Jensen LT, Zerbe O, Winge DR and Vaák M: Engineering of metallothionein-3 neuroinhibitory activity into the inactive isoform metallothionein-1. J Biol Chem 277: 37023 37028, 2002. PMID: 12130647. DOI:10.1074/jbc.M205730200

24 Dziegiel P: Expression of metallothioneins in tumor cells. Pol J Pathol 55: 3-12, 2004. PMID: 15195701.

25 Pastuszewski W, Dziegiel P, Krecicki T, Podhorska-Okolow M, Ciesielska U, Gorzynska E and Zabel M: Prognostic significance of metallothionein, p53 protein and Ki-67 antigen expression in laryngeal cancer. Anticancer Res 27: 335-342, 2007. PMID: 17352251

26 Whitfield ML, George LK, Grant GD and Perou CM: Common markers of proliferation. Nat Rev Cancer 6: 99-106, 2006. PMID: 16491069 . DOI: 10.1038/nrc1802

27 Hiraiwa A, Fujita M, Nagasaka T, Adachi A, Ohashi M and Ishibashi M: Immunolocalization of hCDC47 protein in normal and neoplastic human tissues and its relation to growth. Int $\mathrm{J}$ cancer 74: 180-184, 1997. PMID: 9133452.

28 Padmanabhan V, Callas P, Philips G, Trainer TD and Beatty BG: DNA replication regulation protein $\mathrm{Mcm} 7$ as a marker of proliferation in prostate cancer. J Clin Pathol 57: 1057-1062, 2004. PMID: 15452160 . DOI: $10.1136 /$ jcp.2004.016436

29 Cai K, Luo Y, Li L and Liu Y: [Expression and significance of MCM2, Ki-67 and Rb protein in laryngeal squamous cell carcinomas]. Lin Chung Er Bi Yan Hou Tou Jing Wai Ke Za Zhi 26: 425-428, 2012. PMID: 22803413.

30 Mukherjee G, Muralidhar B, Bafna UD, Laskey RA and Coleman N: MCM immunocytochemistry as a first line cervical screening test in developing countries: a prospective cohort study in a regional cancer centre in India. Br J Cancer 96: 11071111, 2007. PMID: 17342084. DOI: 10.1038/sj.bjc.6603679

31 Stoeber K, Swinn R, Prevost AT, de Clive-Lowe P, Halsall I, Dilworth SM, Marr J, Turner WH, Bullock N, Doble A, Hales $\mathrm{CN}$ and Williams GH: Diagnosis of genito-urinary tract cancer by detection of minichromosome maintenance 5 protein in urine sediments. J Natl Cancer Inst 94: 1071-1079, 2002. PMID: 12122098
32 Zakaria SH, Farag HA and Khater DS: Immunohistochemical Expression of MCM-2 in Oral Epithelial Dysplasias. Appl Immunohistochem Mol Morphol 00: 3-7, 2016. PMID: 26990745. DOI: 10.1097/PAI.0000000000000330

33 Scott IS, Odell E, Chatrath P, Morris LS, Davies RJ, Vowler SL, Laskey RA and Coleman N: A minimally invasive immunocytochemical approach to early detection of oral squamous cell carcinoma and dysplasia. Br J Cancer 94: 1170-1175, 2006. PMID: 16622441. DOI: 10.1038/sj.bjc.6603066

34 Werynska B, Pula B, Muszczynska-Bernhard B, Piotrowska A, Jethon A, Podhorska-Okolow M, Dziegiel P and Jankowska R: Correlation between expression of metallothionein and expression of Ki-67 and MCM-2 proliferation markers in nonsmall cell lung cancer. Anticancer Res 31: 2833-2839, 2011. PMID: 21868526.

35 Han J, Lian M, Fang J, Liu H, Wang R, Zhai J, Fan Yang Y, Fen L, Shi Q, Zhi Ma H, Sun J and Ma Z: Minichromosome maintenance (MCM) protein 4 overexpression is a potential prognostic marker for laryngeal squamous cell carcinoma. $\mathbf{J}$ BUON 22: 1272-1277. PMID: 29135113.

36 Pierzchała P, Pasz-Walczak G and Jeziorski A: New prognostic factors in breast cancer; review of the literature. Wspolczesna Onkol 8: 429-434, 2004.

37 Bukholm IRK, Bukholm G, Holm R and Nesland JM: Association between histology grade, expression of HsMCM2, and cyclin A in human invasive breast carcinomas. J Clin Pathol 56: 368-373, 2003. PMID: 12719458.

38 Kametaka A, Takagi M, Hayakawa T, Haraguchi T, Hiraoka Y and Yoneda Y: Interaction of the chromatin compaction-inducing domain (LR domain) of Ki-67 antigen with HP1 proteins. Genes Cells 7: 1231-1242, 2002. PMID: 12485163.

39 Scholzen T, Endl E, Wohlenberg C, van der Sar S, Cowell IG, Gerdes $\mathrm{J}$ and Singh PB: The Ki-67 protein interacts with members of the heterochromatin protein 1 (HP1) family: a potential role in the regulation of higher-order chromatin structure. J Pathol 196: 135-144, 2002. PMID: 12485163.

40 Endl E, Kausch I, Baack M, Knippers R, Gerdes J and Scholzen T: The expression of Ki-67, MCM3, and p27 defines distinct subsets of proliferating, resting, and differentiated cells. J Pathol 195: 457-462, 2001. PMID: 11745678. DOI: 10.1002/path.978

Received March 20, 2019

Revised April 11, 2019

Accepted April 12, 2019 